\title{
HLA-DR-DQhaplotypes and specificity of the initial autoantibody in islet specific autoimmunity
}

\section{Finnish Pediat Diabet Register}

2020-11

Finnish Pediat Diabet Register, Mikk, M-L , Pfeiffer , S , Kiviniemi , M , Laine , A-P , Lempainen , J , Härkönen , T , Toppari , J , Veijola , R , Knip , M \& Ilonen , J 2020 , ' HLA-DR-DQhaplotypes and specificity of the initial autoantibody in islet specific autoimmunity ' , Pediatric Diabetes , vol. 21 , no. 7 , pp. 1218-1226 . https://doi.org/10.1111/pedi.13073

http://hdl.handle.net/10138/331979

https://doi.org/10.1111/pedi.13073

acceptedVersion

Downloaded from Helda, University of Helsinki institutional repository.

This is an electronic reprint of the original article.

This reprint may differ from the original in pagination and typographic detail.

Please cite the original version. 
HLA-DR-DQ haplotypes and specificity of the initial autoantibody in islet specific autoimmunity

Short running title: HLA and the first antibody in islet autoimmunity

Mari-Liis Mikk ${ }^{1}$, Sophie Pfeiffer ${ }^{1}$, Minna Kiviniemi ${ }^{1}$, Antti-Pekka Laine ${ }^{1}$, Johanna Lempainen ${ }^{1,2,3}$, Taina Härkönen ${ }^{4,5}$, Jorma Toppari ${ }^{2,6}$, Riitta Veijola ${ }^{7,8}$, Mikael Knip ${ }^{4,5,9,10}$, Jorma Ilonen ${ }^{1}$, the Finnish Pediatric Diabetes Register

${ }^{1}$ Immunogenetics Laboratory, Institute of Biomedicine, University of Turku, Turku, Finland; ${ }^{2}$ Department of Pediatrics, University of Turku and Turku University Hospital, Turku; ${ }^{3} \mathrm{Clinical}$ Microbiology, Turku University Hospital, Turku. Finland; ${ }^{4}$ Pediatric Research Center, Children Hospital, University of Helsinki and Helsinki University Hospital, Helsinki. Finland; ${ }^{5}$ Research Program for Clinical and Molecular Metabolism, Faculty of Medicine, University of Helsinki, Helsinki, Finland; ${ }^{6}$ Research Centre for Integrative Physiology and Pharmacology, Institute of Biomedicine, University of Turku, Turku, Finland; ${ }^{7}$ Department of Pediatrics, PEDEGO Research Unit, Medical Research Center, University of Oulu, Oulu. Finland; ${ }^{8}$ Department of Children and Adolescents, Oulu University Hospital, Oulu, Finland; ${ }^{9}$ Folkhälsan Research Center, Helsinki, Finland; ${ }^{10}$ Tampere Center for Child Health Research, Tampere University Hospital, Tampere, Finland

\section{Correspondence}

Jorma Ilonen, Immunogenetics Laboratory, University of Turku, FI-20014 Turku, Finland. E-mail: jorma.ilonen@utu.fi

\section{Funding information} ademy of Finland (Decision No. 286765), Sigrid Jusélius Foundation, JDRF

\section{Conflict of interest}

The authors have no conflicts of interest to report.

This article has been accepted for publication and undergone full peer review but has not been through the copyediting, typesetting, pagination and proofreading process which may lead to differences between this version and the Version of Record. Please cite this article as doi: $10.1111 /$ pedi.13073 


\section{Abstract}

Objective: We aimed to clarify the association of various HLA risk alleles with different types of autoantibodies initiating islet specific autoimmunity.

Methods: Follow-up cohorts from the Finnish Type 1 Diabetes Prediction and Prevention (DIPP) study and children diagnosed with type 1 diabetes from the Finnish Pediatric Diabetes Register (FPDR) were analyzed for the presence of autoantibodies to insulin (IAA), glutamic acid decarboxylase (GADA), IA-2 antigen (IA-2A) and zinc transporter 8 (ZnT8A) and genotyped for HLA DR/DQ alleles. In the DIPP study autoantibodies were regularly analyzed from birth up to 15 years of age.

Results: In the DIPP cohort 621 children developed one single persistent autoantibody, GADA in 284, IAA in 268 and IA-2A in 40 cases. Highly significant differences in the specificity of the first autoantibody were observed between HLA genotypes. Homozygotes for the DR3-DQ2 haplotype had almost exclusively GADA as the first autoantibody whereas a more even distribution between GADA and IAA was found in DR3-DQ2/DR4-DQ8 as well as DR3-DQ/ $x$ and DR4-DQ8/ $x$ genotypes ( $x$ referring to neutral haplotypes). In DR4-DQ8 positive genotypes with the DRB1*04:01 allele IAA was more often the first autoantibody than in DRB1*04:04 positive genotypes. Various neutral haplotypes also significantly affected the relative proportions of different initial autoantibodies. These findings were confirmed and expanded in a series of 1591 T1D children under the age of 10 years from FPDR.

Conclusions: These results emphasize the importance of HLA class II polymorphisms in the recognition of autoantigen epitopes in the initiation of various pathways of the autoimmune -sponse.

KEY WORDS: type 1 diabetes, HLA genotypes, islet specific autoantibodies 


\section{INTRODUCTION}

Susceptibility to type 1 diabetes (T1D) is strongly associated with polymorphisms in HLA class II genes. Various alleles especially in HLA-DR and -DQ loci and their specific combinations are associated either with an increased risk or protection against disease development ${ }^{1,2}$. Immune responses including islet specific autoimmunity are initiated when CD4+ T cells recognize specific antigen epitopes bound by HLA class II molecules on the surface of antigen presenting cells. The allelic variability in the capacity to bind and present various critical peptide epitopes most probably explains the disease associations between HLA alleles and multiple diseases ${ }^{3}$.

Heterogeneity in the pathogenic mechanisms of T1D has long been suspected based on the association with two major HLA class II haplotypes ${ }^{4}$. HLA DR3-DQ2 and DR4-DQ8 are both associated with disease risk and the genotype combining both haplotypes confers a strongly increased risk higher than homozygosity for either haplotype ${ }^{2}$. Recent follow-up studies of children with genetic susceptibility to type 1 diabetes have also supported heterogeneity. In most cases either insulin autoantibodies (IAA) or autoantibodies specific for glutamic acid decarboxylase 65 (GADA) are the first autoantibodies to appear reflecting the development of islet specific autoimmunity. Subjects with IAA as the initial autoantibody are more often positive for the HLA DR4-DQ8 haplotype and the T1D associated INS gene genotype and the peak age at the seroconversion is in early childhood before the age of 2 years whereas the appearance of GADA

arts usually later in childhood and continues at higher level up to the adulthood. GADA-initiated autoimmunity is typically associated with the HLA DR3-DQ2 haplotype and is not associated with the presence of INS risk genotype but instead T1D associated alleles within the IKZF4/ERBB3 gene region $^{5,6}$. These differences suggest heterogeneity in the pathogenetic processes and environmental influences triggering islet autoimmunity and $\beta$-cell destruction.

In this study we wanted to further characterize the diversity of HLA associations between children developing different islet specific autoantibodies initializing the autoimmune process by analyzing HLA DR-DQ genotypes including variations between various DR4 subtypes in DR4-DQ8 
haplotypes and various "neutral" HLA-DR-DQ haplotypes in children followed in the Finnish Diabetes Prediction and Prevention (DIPP) study ${ }^{7}$. The obtained results were further confirmed and expanded in the series of recently diagnosed children from the Finnish Pediatric Diabetes Register (FPDR) who at diagnosis had autoantibody combinations strongly suggesting presence of either IAA, GADA or IA-2A as the first single autoantibody at the initiation of autoimmunity ${ }^{8}$.

\section{METHODS}

\subsection{Study subjects}

The study subjects were recruited in the Finnish DIPP (ClinicalTrials.gov Identifier: NCT03269084) and FPDR ${ }^{9}$ studies. A written informed consent was required for all participants from a parent or legal guardian. The Ethics Committees at the three participating University Hospitals, Turku, Oulu and Tampere in DIPP study and The Ethics Committee of the Hospital District of Helsinki and Uusimaa in FPDR have approved the study protocols.

Two separate cohorts with somewhat different eligibility criteria and length of follow-up were analyzed in the DIPP follow-up study. The first cohort was recruited from November 1994 to August 2004 and included 9297 children with at least three visits for the measurements of autoantibodies. Among them there were 497 children who had only one specific autoantibody r resent at the initiation of persistent islet specific autoimmunity. Originally all children with $D Q B 1 * 02 / D Q B 1^{*} 03: 02$ and $D Q B 1^{*} 03: 02 / x$ ( $x$ any allele except $D Q B 1^{*} 02, D Q B 1 * 03: 01$ or $D Q B 1 * 06: 02 / 03)$ genotypes were eligible but beginning from April $1997 \mathrm{DQB1}$ 06:02 and DQB1*06:03 alleles were differentiated and children with $D Q B 1 * 03: 02 / D Q B 1 * 06: 02$ were excluded but those with $D Q B 1^{*} 03: 02 / D Q B 1^{*} 06: 03$ were eligible. In one of the three centers also boys with the $D Q A 1^{*} 05-D Q B 1 * 02 / x$ (x any allele except $D Q B 1^{*} 03: 01, D Q B 1 * 03: 02, D Q B 1 * 06: 02$, $D Q B 1 * 06: 03$ or $D Q A 1 * 02: 01-D Q B 1 * 02$ combination) genotype were recruited since January 1998 . 
Another DIPP cohort was recruited from March 2010 to the end of year 2015 included 3377 children with at least three visits for the analysis of autoantibodies, and persistent islet specific autoimmunity with only one specific autoantibody present was detected in 124 children. Eligible genotypes included combinations of HLA DQA1*05-DQB1*02 (DR3-DQ2) and $D R B 1 * 04: 01 / 02 / 04 / 05-D Q A 1 * 03-D Q B 1 * 03: 02$ haplotypes (DR4-DQ8), homozygosity for DR3-DQ2 or DR4-DQ8 haplotypes as well as DRB1*04:01/02/05-DQA1*03-DQB1*03:02 in combinations with either the $D Q B 1{ }^{*} 05: 01$ (DR1-DQ5), DQA1*02:01-DQB1*02 (DR7-DQ2), DQB1*04 (DR8-DQ4), (DQA1*03-DQB1*03:03 (DR9-DQ9) or DQB1*06:04 (DR13-DQ6.4) haplotype and the DR3DQ2/DR9-DQ9 combination.

Both cohorts were followed for the appearance of autoantibodies either until to the age of 15 years or until April 2017. In addition to these cohorts we analyzed 3355 children from the Finnish Pediatric Diabetes Register (FPDR) who had been diagnosed with T1D before the age of 10 years and who had been tested for the presence of islet autoantibodies and genotyped for HLA-DR/DQ loci. Autoantibody patterns suggesting presence of either IAA, GADA or IA-2A as the first autoantibody were identified in 1559 children (46.5\%). These patterns were observed to associate with either insulin, GADA or IA-2A being the first autoantibody initializing autoimmunity in the earlier follow-up study of 128 children in the DIPP project who were followed from birth up to the diagnosis of T1D ${ }^{8}$. As IAA and GADA were the two most common autoantibodies to appear as the first autoantibody $(106 / 128,82.8 \%)$, IAA could be deduced to be usually the first autoantibody detected in cases where IAA was present at diagnosis but not GADA, and similarly GADA without inA could be deduced in most cases to be the first autoantibody and this was also confirmed in the study. IA-2A was found to be the first autoantibody in 13 (10.2\%) cases and ZnT8A in nine (7.0\%) cases. In seven (53.8\%) of the 13 cases in whom IA-2A was the first autoantibody it was also the only autoantibody present at diagnosis. Although IA-2A present as the only autoantibody at diagnosis implied that that antibody was the first autoantibody detected, this association was weaker than the associations in autoantibody combinations suggesting presence of IAA or GADA as the first autoantibody. Due to the increasing number of inverse seroconversions by increasing 
follow-up time especially in the group with IAA as the first autoantibody we decided to include only children diagnosed before the age of 10 years into the analysis.

\subsection{HLA typing}

All available autoantibody positive samples in the DIPP follow-up cohort and samples of the FPDR were analyzed for HLA-DR/DQ haplotypes present using a step-wise method starting with DQB1 alleles and continuing with DQA1 when informative and also with HLA-DR4 subtyping if relevant. This typing procedure based on various procedures using sequence specific lanthanide-labelled oligonucleotides defines major DR/DQ haplotypes found in populations of European ancestry and differentiates DRB1, DQA1 and DQB1 loci at four digit level when relevant for T1D risk ${ }^{10}$.

\subsection{Autoantibody analysis}

In the DIPP cohort serum samples were originally screened for islet cell autoantibodies (ICA) using immunofluorescence staining of human pancreas preparations and in the case of ICA positivity, all samples from such subjects were studied for IAA, GADA and IA-2A using radio-binding assays. Since year 2003, these three biochemical autoantibodies were used for primary screening. In audition, all sera from the first 1004 enrolled DIPP children born between November 1994 and July 1997 were analyzed for these three biochemical autoantibodies. In the earlier DIPP cohort, sera from subjects positive for IAA, GADA and/or IA-2A were also analyzed for ZnT8A autoantibodies.

In the FPDR, sera taken at the diagnosis (from 2 months before until 2 weeks after the diagnosis date) of children were analyzed for IAA, GADA, IA-2A and ZnT8A. Similar assays for autoantibody detection were used in both DIPP and FPDR samples but the analyses for IAA, GADA and IA-2A were carried out in different laboratories and thus slightly different cut-off values were 
used. Details of the assays are published earlier as well as the results of these laboratories in autoantibody standardization programs ${ }^{11-14}$.

\subsection{Statistical analysis}

Chi-square test of independence was used in the comparison of groups with various HLA genotypes and first islet specific autoantibodies. Spreadsheet program used for analyzing the distribution was obtained from the address:

http://www.biostathandbook.com/chiind.html (The Handbook of Biological Statistics, John H. McDonald (C2014). Yates' correction was performed in two-way tables when appropriate using https://statpages.info/ctab2x2.html program.

\section{RESULTS}

\subsection{Follow-up cohorts in the DIPP study}

m iotal of 497 children in the DIPP cohort recruited from November 1994 to September 2004 had developed a single first persistent autoantibody, a majority of them either GADA or IAA. IA-2A and ZnT8A were relatively rare as the first autoantibodies. However, ZnT8A was measured only from subjects who were also positive for some other biochemical autoantibodies and thus the number would be apparently higher if all children had been tested.

When the distribution of the first autoantibodies initializing islet autoimmunity were compared between children with different major HLA risk genotypes based on the presence of the DR3-DQ2 and/or DR4-DQ8 haplotypes highly significant differences were detected (Figure 1A, 
$P=0.000019, \mathrm{df}=12)$. GADA was found as the first autoantibody in five (83\%) of six children homozygous for DR3-DQ2 and GADA was the most common first autoantibody also among those with the DR3-DQ2/DR4-DQ8 (52.2\%) and DR4-DQ8/DR4-DQ8 (59.3\%) genotypes, whereas IAA was most often the first autoantibody among those with DR3-DQ2/x (36.8\%) and DR4-DQ8/x genotypes (47.2\%) genotypes. IA-2A was rarely the first autoantibody. It was most common in DR4-DQ8 homozygotes and in those carrying DR3-DQ2/x genotype albeit the numbers were small. The later DIPP cohort recruited since March 2010 was similarly analyzed for the appearance of various islet specific autoantibodies in different HLA genotypes. In this cohort eligibility criteria were somewhat different and mean follow-up time shorter than in the old cohort and ZnT8 autoantibody results were not available from this cohort. Thus, the analyses of two cohorts were made separately. Also in this cohort a highly significant difference was found between the major HLA risk genotypes (Figure $1 b, P=.000067, \mathrm{df}=8$ ). Recruitment of DR3-DQ2 homozygotes in all centers during this period increased their numbers and confirmed the finding of the very strong association of this genotype with GADA as the first autoantibody. In contrast, in both cohorts children with the DR3-DQ2/x genotype (which in fact was just the DR3-DQ2/DR9-DQ9 genotype in the later cohort) were not dominated by GADA initiated autoimmunity like in DR3-DQ2 homozygotes.

Children with the DR4-DQ8/x genotype in both cohorts had IAA as the most common first autoantibody and especially in the newer cohort the dominance of IAA was obvious. This is probably caused by the fact that in the newer cohort only children positive for DRB1*04:01/2/5 aneles in the DR4-DQ8 haplotypes (mainly DRB1*04:01) were eligible in this group. In the earlier cohort comparison of children with either DRB1*04:01 or DRB1*04:04 positive DR4-DQ8 haplotypes in the DR4-DQ8/x genotype demonstrated that in $D R B 1^{*} 04: 01$ positive children IAA was more often the first autoantibody, whereas in children with $D R B 1 * 04: 04$ positive genotypes GADA was most often the first autoantibody $(P=.0073, \mathrm{df}=2$, Table 1$)$. When various combinations of $D R B 1 * 04: 01$ and $D R B 1 * 04: 04$ were compared among homozygous DR4-DQ8 positive subjects from combined cohorts IAA was the first autoantibody in ten of 27 children with $D R B 1^{*}$ 04:01 homozygosity whereas it was found only in four of 34 cases homozygous for 
$D R B 1 * 04: 04$ or with the $D R B 1^{*} 04: 01 / D R B 1 * 04: 04$ genotype $(P=.043)$ emphasizing the association of IAA with the DRB1*04:01-DQB1*03:02 haplotype.

\subsection{Antibody patterns at the diagnosis of type 1 diabetes in children below the age of 10 years}

Autoantibody data at the diagnosis of T1D were available from 3355 children who were diagnosed below the age of 10 years in FPDR. Autoantibody patterns at the diagnosis suggested presence of either GADA, IAA or IA-2A as the first autoantibody in 1559 of them ${ }^{8}$. Comparison of these groups for the main HLA-DR/DQ genotypes confirmed the strong heterogeneity $(P=8.5 E-12, d f=10)$ and the clear association of the DR3-DQ2/x genotype in addition to DR3-DQ2/DR3-DQ2 homozygosity with the "GADA group" (Figure 2). The proportion of autoantibody combinations suggesting IAA as the first autoantibody was highest among DR4-DQ8 positive genotypes including DR3-DQ2/DR4DQ8, DR4-DQ8/DR4-DQ8 and DR4-DQ8/ $x$ although GADA was still the most common implicated first autoantibody in all of them. IA-2A alone indicating that antibody as the first autoantibody was especially rare among children homozygous for DR3-DQ2 and with DR3-DQ2/x genotype. The genotype group $\mathrm{x} / \mathrm{x}$ without either DR3-DQ2 or DR4-DQ8 lacking from the follow-up cohorts showed their strongest association with GADA initiated autoimmunity but in addition also IAA and IA-2A were relatively common as first autoantibodies compared to DR3-DQ2/ $x$ and DR3-DQ2 homozygous groups.

Further comparisons were performed only between children with autoantibody combinations suggesting either GADA or IAA as the first autoantibody because of the small number of children with IA-2A alone. Preferential association of DRB1*04:01 with IAA-initiated and DRB1*04:04 with GADA-initiated autoimmunity was apparent also when various DR4 subtypes were compared within homozygous DR4-DQ8 genotypes as well as within the DR3-DQ2/DR4-DQ8 and DR4-DQ8/x genotypes (Table 2).

For the analysis of the effect of various neutral or protective haplotypes in combination with either DR3-DQ2 or two different DR4 alleles in DR4-DQ8/x genotypes only those genotypes with at 
least five cases were included. Significant heterogeneity was detected among the carriers of the most frequent $D R B 1^{*} 04: 01$ positive $D R 4-D Q 8 / x$ genotype $(P=.000083, \mathrm{df}=7)$ where DR13-DQ6.3, DR8-DQ4, DR13-DQ6.4 were more often associated with IAA than GADA as the first autoantibody whereas DR1/10-DQ5, DR4-DQ7, DR5-DQ7 and DR7-DQ2 were most often observed with GADA initiated autoimmunity (Table 3). Although no significant differences were detected in the distribution of neutral haplotypes between IAA and GADA initiated autoimmunity in the DR3$\mathrm{DQ} 2 / x$ and DRB1*04:04 positive DR4/x genotype, a tendency for a similar type of preferential association was seen (Table 3).

Table 4 shows the associations of implicated GADA and IAA initiated autoantibody responses to genotypes where only neutral or protective haplotypes were present without any DR3-DQ2 or DR4-DQ8 haplotypes. Though relatively rare, we identified 191 children with such combinations in the FPDR series. There was a tendency of heterogeneity also among these genotypes. The antibody combinations implicating GADA as the first autoantibody were most common in all genotypes but the proportion of IAA was relatively high in genotypes where DR1/10-DQB1*05 was associated with DR4-DQ7 and DR8-DQ4 as well as in DR13-DQ6.4/DR8-DQ4 and DR7-DQ2/DR8DQ4 genotypes.

\section{DISCUSSION}

e preferential association of IAA positivity with HLA DR4-DQ8 and GADA with HLA DR3-DQ2 haplotype at diagnosis of T1D has been known for decades ${ }^{15-18}$ but follow-up studies have recently demonstrated that the association is even more strong when the first autoantibody initiating islet autoimmunity is considered ${ }^{6,19}$. This strong association agrees with the role of class II molecules in antigen presentation where differences in amino acids between various alleles affect the ability of different peptide epitopes to be bound and be presented to CD4+ T cells initiating the immune response $^{20}$. 
Earlier studies have shown a high incidence of IAA-initiated autoimmunity in DR4-DQ8 homozygous children and GADA-initiated autoimmunity in DR3-DQ2 homozygous children with a more even picture in children with the heterozygous DR3-DQ2/DR4-DQ8 genotype ${ }^{6,19}$. Most conspicuous was the dominance of GADA as the first autoantibody in DR3-DQ2 homozygotes, whereas in the group of DR4-DQ8 homozygotes also GADA was relatively frequently the first autoantibody. In the TEDDY study also a group of children with DR4-DQ8/DR8-DQ4 genotype was included with a strong dominance of IAA-initiated autoimmunity ${ }^{6}$. We now analyzed in the DIPP study various DR4 subtypes in DR4-DQ8 haplotypes which also differ for the strength of their association with $\mathrm{T}_{1} \mathrm{D}^{1,21}$. There was a clear difference between the two common DR4 alleles in Finland, DRB1*04:01 was more strongly associated with IAA initiated autoimmunity than $D R B 1^{*}$ 04:04 which was preferentially found among children with GADA as the first autoantibody.

The difference was most pronounced when comparing $D R B 1^{*} 04: 01$ homozygous and $D R B 1 * 04: 04$ homozygous children but similarly detected in comparison of the DR4 subtype in DR3-DQ2/DR4-DQ8 heterozygotes and in the DR4-DQ8/x genotype. Differences between various DR4 subtypes in preferential autoantigen recognition are in accordance with findings where islet autoantigen epitopes have been presented to specific CD4+ T cell by either DRB1*04:01 ${ }^{22}$ or DB1*04:04 ${ }^{23}$ alleles but also by both of these alleles ${ }^{24}$. An important difference between these two alleles is also seen in the probability to develop autoantibodies against 21-OH hydroxylase and Addison's disease detected together with T1D in autoimmune polyendocrine syndrome 2 . Besides the major DR3-DQ2 association, antibodies to 21- hydroxylase and Addison's disease are associated only with DRB1*04:04 and not with the DRB1*04:01 positive DR4-DQ8 haplotypes despite stronger association of T1D with DRB1*04:0125,26

Due to the strong linkage between DR and DQ loci the actual molecules responsible for the initiation of autoimmunity reflected in circulating autoantibodies is not easy to define but our observation on the importance of DR4 subtypes in DR4-DQ8 haplotypes supports the importance of also DR molecules besides DQ similarly to the known genetic associations of alleles in both molecules. 
In addition to the class II molecules in the known risk associated DR3-DQ2 and DR4-DQ8 haplotypes, neutral haplotypes affected the specificity of the first autoantibody to appear. This was demonstrated by the increase of IAA-initiated autoantibody response in subjects with a genotype where DR3-DQ2 was associated with some of these neutral haplotypes compared to the nearly exclusive GADA-initiated autoimmunity in DR3-DQ2 homozygotes. When genotypes combining various neutral haplotypes with either DR3-DQ2 or DR4-DQ8 risk haplotypes were compared at diagnosis for autoantibody combinations implicating either IAA or GADA as the initial autoantibody the strongest differences were observed in the largest group with DRB1*0401 positive DR4-DQ8/x genotypes but similar tendencies of IAA or GADA associations were also seen in DRB1*04:04 positive DR4-DQ8/ $x$ and DR3-DQ2/ $x$ genotypes as well as in genotypes where only neutral or slightly protective haplotypes were present although the number of children with numerous different genotype combinations was too low for any definitive results.

There are limitations of the current study. ZnT8 autoantibodies were analyzed only in children testing positive for other islet specific autoantibodies in the first DIPP follow-up cohort and not at all in the later DIPP cohort. It was anyway rare in subjects who developed also other autoantibodies which is the case for the majority of cases eventually developing T1D. Similar results obtained in both DIPP follow-up cohorts for the differences in genetic associations of major IAA- and GADA-initiated groups also indicate that ZnT8A is relatively rare as initial autoantibody in islet specific autoimmunity. Associations of IAA and GADA initiated autoantibody responses with different major HLA risk genotypes, importantly including DR4 subtypes, were also supported by we data obtained from autoantibody combinations at diagnosis suggesting either IAA or GADA being the first islet specific autoantibody. These autoantibody combinations can be, however, identified only in less than half of children diagnosed with T1D and because the tendency of IAA to disappear with the increasing duration of the follow-up, the possibility for deduction is limited to the youngest age group. Sera obtained at diagnosis of T1D are easily available in high numbers compared to follow-up studies starting in early childhood and may also supply genotypes less common in T1D, which are not selected in genetic screening for follow-up studies. However, more comparisons of initial autoantibodies and autoantibodies at diagnosis are still needed from follow- 
up studies. The results obtained anyway demonstrate that based on autoantibody analyses at diagnosis a large proportion of prepubertal cases can be identified with a reasonable accuracy to have either IAA- or GADA-initiated autoimmunity. These "endotypes" have strong associations with different HLA risk genotypes and using various sample series and information collected at diagnosis can produce more information on genetics and immunological features as well as demography and epidemiology associated with these two major forms of islet specific autoimmunity in childhood.

In conclusion, the strong association between the specificity of first islet autoantibody to appear and the HLA class II genotypes supports the hypothesis that recognition of a limited number of autoantigen epitopes is crucial in the initiation of the pathogenic process and may furthermore explain specific class II HLA associations with possible environmental effects triggering the disease process.

\section{ACKNOWLEDGEMENTS}

The authors thank the dedicated personnel of the Finnish Type 1 DIPP Study and the Finnish Pediatric Diabetes Register as well as the participating children and their families. The study was supported by the Academy of Finland (Decision No. 286765) the Sigrid Jusélius Foundation and JDRF.

\section{REFERENCES}

1. Erlich H, Valdes AM, Noble J, et al. HLA DR-DQ haplotypes and genotypes and type 1 diabetes risk: analysis of the type 1 diabetes genetics consortium families. Diabetes. 2008;57(4):1084-1092. 
2. Thomson G, Valdes AM, Noble JA, et al. Relative predispositional effects of HLA class II DRB1-DQB1 haplotypes and genotypes on type 1 diabetes: a meta-analysis. Tissue Antigens. 2007;70(2):110-127.

3. Howell WM. HLA and disease: guilt by association. Int J Immunogenet. 2014;41(1):1-12.

4. Ludvigsson J, Samuelsson U, Beauforts C, et al. HLA-DR 3 is associated with a more slowly progressive form of type 1 (insulin-dependent) diabetes. Diabetologia. 1986;29(4):207-210.

5. Ilonen J, Hammais A, Laine AP, et al. Patterns of $\beta$-cell autoantibody appearance and genetic associations during the first years of life. Diabetes. 2013;62(10):3636-3640.

6. Krischer JP, Lynch KF, Schatz DA, et al. The 6 year incidence of diabetes-associated autoantibodies in genetically at-risk children: the TEDDY study. Diabetologia. 2015;58(5):980-987.

7. Bauer W, Veijola R, Lempainen J, et al. Age at Seroconversion, HLA Genotype, and Specificity of Autoantibodies in Progression of Islet Autoimmunity in Childhood. J Clin Endocrinol Metab. 2019;104(10):4521-4530.

8. Ilonen J, Lempainen J, Hammais A, et al. Primary islet autoantibody at initial seroconversion and autoantibodies at diagnosis of type 1 diabetes as markers of disease heterogeneity. Pediatr Diabetes. 2018;19(2):284-292.

9. Parkkola A, Härkönen T, Ryhänen SJ, Ilonen J, Knip M, the Finnish Pediatric Diabretes Regisdter. Extended family history of type 1 diabetes and phenotype and genotype of newly diagnosed children. Diabetes Care 2013;36:348-354

10. Ilonen J, Kiviniemi M, Lempainen J, et al. Genetic susceptibility to type 1 diabetes in childhood - estimation of HLA class II associated disease risk and class II effect in various phases of islet autoimmunity. Pediatr Diabetes. 2016;17 Suppl 22:8-16.

11. Juusola M, Parkkola A, Härkönen T, et al. Positivity for Zinc Transporter 8 Autoantibodies at Diagnosis Is Subsequently Associated With Reduced $\beta$-Cell Function and Higher Exogenous Insulin Requirement in Children and Adolescents With Type 1 Diabetes. Diabetes Care. 2016;39(1):118-121.

12. Turtinen M, Härkönen T, Parkkola A, llonen J, Knip M, Finnish Pediatric Diabetes Register. Sex as a determinant of type 1 diabetes at diagnosis. Pediatr Diabetes. 2018;19(7):12211228.

Siljander HT, Simell S, Hekkala A, et al. Predictive characteristics of diabetes-associated autoantibodies among children with HLA-conferred disease susceptibility in the general population. Diabetes. 2009;58(12):2835-2842.

14. Pöllänen PM, Lempainen J, Laine AP, et al. Characterisation of rapid progressors to type 1 diabetes among children with HLA-conferred disease susceptibility. Diabetologia. 2017;60(7):1284-1293.

15. Vandewalle CL, Decraene T, Schuit FC, De Leeuw IH, Pipeleers DG, Gorus FK. Insulin autoantibodies and high titre islet cell antibodies are preferentially associated with the HLA DQA1*0301-DQB1*0302 haplotype at clinical type 1 (insulin-dependent) diabetes mellitus before age 10 years, but not at onset between age 10 and 40 years. The Belgian Diabetes Registry. Diabetologia. 1993;36(11):1155-1162. 
16. Ziegler R, Alper CA, Awdeh ZL, et al. Specific association of HLA-DR4 with increased prevalence and level of insulin autoantibodies in first-degree relatives of patients with type I diabetes. Diabetes. 1991;40(6):709-714.

17. Sanjeevi CB, Falorni A, Kockum I, Hagopian WA, Lernmark A. HLA and glutamic acid decarboxylase in human insulin-dependent diabetes mellitus. Diabet Med. 1996;13(3):209217.

18. Vandewalle CL, Falorni A, Lernmark A, et al. Associations of GAD65- and IA-2autoantibodies with genetic risk markers in new-onset IDDM patients and their siblings. The Belgian Diabetes Registry. Diabetes Care. 1997;20(10):1547-1552.

19. Giannopoulou EZ, Winkler C, Chmiel R, et al. Islet autoantibody phenotypes and incidence in children at increased risk for type 1 diabetes. Diabetologia. 2015;58(10):2317-2323.

20. Klein J, Sato A. The HLA system. First of two parts. N Engl J Med. 2000;343(10):702-709.

21. Noble JA, Valdes AM, Cook M, Klitz W, Thomson G, Erlich HA. The role of HLA class II genes in insulin-dependent diabetes mellitus: molecular analysis of 180 Caucasian, multiplex families. Am J Hum Genet. 1996;59(5):1134-1148.

22. Endl J, Otto $H$, Jung $G$, et al. Identification of naturally processed T cell epitopes from glutamic acid decarboxylase presented in the context of HLA-DR alleles by T lymphocytes of recent onset IDDM patients. J Clin Invest. 1997;99(10):2405-2415.

23. Mannering SI, Pang SH, Williamson NA, et al. The A-chain of insulin is a hot-spot for CD4+ T cell epitopes in human type 1 diabetes. Clin Exp Immunol. 2009;156(2):226-231.

24. Mannering SI, Harrison LC, Williamson NA, et al. The insulin A-chain epitope recognized by human T cells is posttranslationally modified. J Exp Med. 2005;202(9):1191-1197.

25. Yu L, Brewer KW, Gates S, et al. DRB1*04 and DQ alleles: expression of 21-hydroxylase autoantibodies and risk of progression to Addison's disease. J Clin Endocrinol Metab. 1999;84(1):328-335.

26. Gombos Z, Hermann R, Kiviniemi M, et al. Analysis of extended human leukocyte antigen haplotype association with Addison's disease in three populations. Eur J Endocrinol. 2007;157(6):757-761. 


\section{APPENDIX}

The Finnish Pediatric Diabetes Register comprises the following investigators:

Principal Investigator: Mikael Knip (Children's Hospital, Helsinki University Hospital) Steering Committee: Per-Henrik Groop (Folkhälsan Research Center), Jorma Ilonen (Immunogenetics Laboratory, University of Turku), Timo Otonkoski (Children's Hospital, Helsinki University Hospital), Riitta Veijola (Department of Pediatrics, Oulu University Hospital). Locally responsible investigators:

Alar Abram (Department of Pediatrics, Kanta-Häme Central Hospital), Henrikka Aito (Department of Pediatrics, HUS Porvoo Hospital), Ivan Arkhipov (Department of Pediatrics, Mehiläinen LänsiPohja Central Hospital), Elina Blanco-Sequeiros (Department of Pediatrics, Central Ostrobothnia Central Hospital), Jonas Bondestam (Department of Pediatrics, HUS Lohja Hospital), Markus Granholm (Department of Pediatrics, Jakobstad Hospital), Maarit Haapalehto-Ikonen (Department of Pediatrics, Rauma Hospital), Torsten Horn (Department of Pediatrics, Central Hospital of Central Finland), Hanna Huopio (Department of Pediatrics, Kuopio University Hospital), Joakim Janer (Department of Pediatrics, HUS Raasepori Hospital), Christian Johansson (Department of Pediatrics, Åland Central Hospital), Liisa Kalliokoski (Department of Pediatrics, Kainuu Central Hospital), Päivi Keskinen (Department of Pediatrics, Tampere University Hospital), Anne Kinnala (Department of Pediatrics, Turku University Central Hospital), Maarit Korteniemi (Department of Pediatrics, Central Hospital of Lapland), Hanne Laakkonen (Department of Pediatrics, HUS Hyvinkää Hospital), Jyrki Lähde (Department of Pediatrics, Satakunta Central Hospital), Päivi Miettinen (HUS New Children's Hospital). Päivi Nykänen (Department of Pediatrics, Mikkeli Central Hospital), Erik Popov (Department of Pediatrics, Vaasa Central Hospital), Mari Pulkkinen (Department of Pediatrics, HUS Jorvi Hospital), Maria Salonen (Department of Pediatrics, Kymenlaakso Central Hospital), Pia Salonen (Department of Pediatrics, Päijät-Häme Central Hospital), Juhani Sankala (Department of Pediatrics, Savonlinna Central Hospital), Virpi Sidoroff (Department of Pediatrics, North Karelia Central Hospital), Anne-Maarit Suomi (Department of Pediatrics, South Ostrobothnia Central Hospital, Tuula Tiainen (Department of Pediatrics, South nurelia Central Hospital), Riitta Veijola (Department of Pediatrics, Oulu University Hospital) 


\section{LEGENDS FOR THE FIGURES}

Figure 1. Distribution of the first autoantibodies initializing islet autoimmunity compared between children with different major HLA risk genotypes based on the presence of DR3-DQ2 and/or DR4DQ8 haplotypes. A significant difference between various HLA genotypes was detected both in the first DIPP follow-up cohort recruited between years 1994-2004 ( $A, P=0.000019$. $d f=12)$ and the second cohort recruited between years 2010-2015 ( $B, P=0.000067, \mathrm{df}=8)$. $\mathrm{ZnT8}$ autoantibodies were measured only in the first cohort and only from subjects positive for IAA, GADA or IA-2A.

Figure 2. Distribution of autoantibody patterns at the diagnosis suggesting presence of either GADA, IAA or IA-2A as the first autoantibody based on the Finnish Pediatric Diabetes Register- Significant difference between various HLA genotypes was detected $(P=8.5 \mathrm{E}-12, \mathrm{df}=10)$. 
Table 1. First single persistent autoantibodies initialising islet specific autoimmunity in DIPP children with the DR4-DQ8/x genotype and either DRB1*04:01 or DRB1*04:04 allele in the HLADR4-DQ8 haplotype. Cohort of children recruited for the study from 1994 to 2004. $x=$ other than the DR3-DQ2 or DR4-DQ8 haplotype. Most common first autoantibody in each genotype marked bold

\begin{tabular}{|l|c|c|c|c|c|c|c|}
\hline \multirow{2}{*}{$\begin{array}{l}\text { DR4 allele in } \\
\text { children with DR4- }\end{array}$} & \multicolumn{2}{|c|}{$\begin{array}{c}\text { GADA } \\
\mathrm{N}=106\end{array}$} & \multicolumn{2}{c|}{$\begin{array}{c}\text { IAA } \\
\mathrm{N}=128\end{array}$} & \multicolumn{2}{c|}{$\begin{array}{c}\text { IA-2A } \\
\mathrm{N}=19\end{array}$} & $\begin{array}{c}\text { Total } \\
\mathrm{N}=253\end{array}$ \\
\cline { 2 - 8 } DQ8 genotypes & $\mathrm{N}$ & $\%$ & $\mathrm{~N}$ & $\%$ & $\mathrm{~N}$ & $\%$ & $\mathrm{~N}$ \\
\hline DRB1*04:01 & 68 & 36.4 & $\mathbf{1 0 2}$ & $\mathbf{5 4 . 5}$ & $\mathbf{1 7}$ & $\mathbf{9 . 1}$ & 187 \\
\hline DRB1*04:04 & $\mathbf{3 8}$ & $\mathbf{5 7 . 6}$ & 26 & 39.4 & 2 & 2.9 & 66 \\
\hline
\end{tabular}

$P=0.0073, \mathrm{df}=2$

GADA vs IAA: $P=0.008, \mathrm{df}=1$

Table 2. Distribution of deduced initial islet specific autoantibodies in children with either DRB1*04:01/x or DRB1*04:04/ $x$ in various HLA-DR4-DQ8 genotypes. Autoantibodies measured from sera at the diagnosis of T1D in the Finnish Pediatric Diabetes Register. Most common deduced first autoantibody in each genotype marked bold

\begin{tabular}{|c|c|c|c|c|c|}
\hline & \multicolumn{5}{|c|}{ Probable initial autoantibody } \\
\hline $\begin{array}{l}\text { DR4-DQ8/DR4-DQ8 } \\
\text { genotypes }\end{array}$ & \multicolumn{2}{|c|}{$\begin{array}{l}\text { GADA } \\
\mathrm{N}=62\end{array}$} & \multicolumn{2}{|c|}{$\begin{array}{c}\text { IAA } \\
\mathrm{N}=48\end{array}$} & $\begin{array}{c}\text { Total } \\
110\end{array}$ \\
\hline DRB1*04 alleles & $\mathrm{N}$ & $\%$ & $\mathrm{~N}$ & $\%$ & $\mathrm{~N}$ \\
\hline$* 04: 01 / * 04: 01$ & 26 & 42.6 & 35 & 57.4 & 61 \\
\hline$* 04: 01 / * 04: 04$ & 27 & 71.1 & 11 & 28.9 & 38 \\
\hline$* 04: 04 / * 04: 04$ & 9 & 81.8 & 2 & 18.2 & 11 \\
\hline \multicolumn{6}{|l|}{$P=0.0043, \mathrm{df}=2$} \\
\hline $\begin{array}{l}\text { R3-DQ2/DR4-DQ8 } \\
\text { genotypes }\end{array}$ & \multicolumn{2}{|c|}{$\begin{array}{l}\text { GADA } \\
\mathrm{N}=187\end{array}$} & \multicolumn{2}{|c|}{$\begin{array}{c}\text { IAA } \\
N=127\end{array}$} & $\begin{array}{c}\text { Total } \\
314\end{array}$ \\
\hline DRB1*04 allele & $\mathrm{N}$ & $\%$ & $\mathrm{~N}$ & $\%$ & $\mathrm{~N}$ \\
\hline DRB1*04:01 & 120 & 54.5 & 100 & 45,5 & 220 \\
\hline DRB1*04:04 & 60 & 71.4 & 24 & 28.6 & 84 \\
\hline \multicolumn{6}{|l|}{$P=0.0074, \mathrm{df}=1$} \\
\hline DR4-DQ8/x genotypes & \multicolumn{2}{|c|}{$\begin{array}{l}\text { GADA } \\
\mathrm{N}=306\end{array}$} & \multicolumn{2}{|c|}{$\begin{array}{c}\text { IAA } \\
N=253\end{array}$} & $\begin{array}{c}\text { Total } \\
559\end{array}$ \\
\hline DRB1*04 alleles & $\mathrm{N}$ & $\%$ & $\mathrm{~N}$ & $\%$ & $\mathrm{~N}$ \\
\hline DRB1*04:01 & 216 & 50.0 & 216 & 50.0 & 432 \\
\hline DRB1*04:04 & 90 & 70.9 & 37 & 29.1 & 127 \\
\hline
\end{tabular}

This article is protected by copyright. All rights reserved. 
Table 3. Distribution of autoantibody combinations associated with various initial islet specific autoantibodies in genotypes where a "neutral" or "slightly protective" haplotype $(X)$ is associated with DR3-DQ2 or DR4-DQ8 haplotypes with either the DRB1*04:01 or DRB1*04:04 subtype. Autoantibodies measured from sera at the diagnosis with T1D in the Finnish Pediatric Diabetes Register. Most common first autoantibody in each genotype marked bold. Only genotypes with a total frequency of at least five are included.

\begin{tabular}{|c|c|c|c|c|c|}
\hline & \multicolumn{5}{|c|}{ Probable initial autoantibody } \\
\hline HLA-genotype & \multicolumn{2}{|c|}{$\begin{array}{l}\text { GADA } \\
\mathrm{N}=145\end{array}$} & \multicolumn{2}{|c|}{$\begin{array}{c}\text { IAA } \\
\mathrm{N}=48\end{array}$} & $\begin{array}{c}\text { Total } \\
\mathrm{N}=193\end{array}$ \\
\hline DR3-DQ2/X & $\mathrm{N}$ & $\%$ & $\mathrm{~N}$ & $\%$ & $\mathrm{~N}$ \\
\hline DR1/10-DQ5 & 69 & 84.1 & 13 & 15.9 & 82 \\
\hline DR4-DQ7 & 5 & 83.3 & 1 & 16.7 & 6 \\
\hline DR5-DQ7 & 7 & 100.0 & 0 & 0.0 & 7 \\
\hline DR7-DQ2 & 4 & 80.0 & 1 & 20.0 & 5 \\
\hline DR13-DQ6.3 & 4 & 66.7 & 2 & 33.3 & 6 \\
\hline DR13-DQ6.4 & 12 & 57.1 & 9 & 42.9 & 21 \\
\hline DR16-DQ5 & 5 & 83.3 & 1 & $16 ., 7$ & 6 \\
\hline DR8-DQ4 & 22 & 71.0 & 9 & 29.0 & 31 \\
\hline DR9-DQ9 & 17 & 58.6 & 12 & 41.4 & 29 \\
\hline \multicolumn{6}{|l|}{$P=0.064, \mathrm{df}=8$} \\
\hline & \multicolumn{2}{|c|}{$\begin{array}{l}\text { GADA } \\
\mathrm{N}=211\end{array}$} & \multicolumn{2}{|c|}{$\begin{array}{c}\text { IAA } \\
N=212\end{array}$} & $\begin{array}{c}\text { Total } \\
\mathrm{N}=423\end{array}$ \\
\hline DR4-DQ8/x: DRB1*04:01 & $\mathbf{N}$ & $\%$ & $\mathrm{~N}$ & $\%$ & $\mathrm{~N}$ \\
\hline DR1/10-DQ5 & 80 & 58.4 & 57 & 41.6 & 137 \\
\hline DR4-DQ7 & 8 & 80.0 & 2 & 20.0 & 10 \\
\hline DR5-DQ7 & 14 & 93.3 & 1 & 6.7 & 15 \\
\hline DR7-DQ2 & 11 & 55.0 & 9 & 45.0 & 20 \\
\hline DR13-DQ6.3 & 13 & 32.5 & 27 & 67.5 & 40 \\
\hline DR13-DQ6.4 & 23 & 47.9 & 25 & 52.1 & 48 \\
\hline DR8-DQ4 & 51 & 38.9 & 80 & 61.1 & 131 \\
\hline DR9-DQ9 & 11 & 50.0 & 11 & 50.0 & 22 \\
\hline \multicolumn{6}{|l|}{$P=0.000083, \mathrm{df}=7$} \\
\hline & \multicolumn{2}{|c|}{$\begin{array}{l}\text { GADA } \\
\mathrm{N}=85\end{array}$} & \multicolumn{2}{|c|}{$\begin{array}{c}\text { IAA } \\
N=36\end{array}$} & $\begin{array}{c}\text { Total } \\
\mathrm{N}=121\end{array}$ \\
\hline DR4-DQ8/x: DRB1*04:04 & $\mathbf{N}$ & $\%$ & $\mathrm{~N}$ & $\%$ & $\mathrm{~N}$ \\
\hline DR1/10-DQ5 & 34 & 73.9 & 12 & 26.1 & 46 \\
\hline DR5-DQ7 & 6 & 85.7 & 1 & 14.3 & 7 \\
\hline DR7-DQ2 & 6 & 85.7 & 1 & 14.3 & 7 \\
\hline DR15-DQ6.2 & 5 & 62.5 & 3 & 37.5 & 8 \\
\hline DR13-DQ6.3 & 4 & 57.1 & 3 & 42.9 & 7 \\
\hline
\end{tabular}




\begin{tabular}{|l|c|c|c|c|c|}
\hline DR13-DQ6.4 & $\mathbf{4}$ & 57.1 & 3 & 42.9 & 7 \\
\hline DR8-DQ4 & $\mathbf{2 0}$ & 62.5 & 12 & 37.5 & 32 \\
\hline DR9-DQ9 & $\mathbf{6}$ & 85.7 & 1 & 14.3 & 7 \\
\hline NS
\end{tabular}

This article is protected by copyright. All rights reserved. 
Table 4. Distribution of autoantibody combinations associated with various initial islet specific autoantibodies in genotypes where only neutral and protective haplotypeps are present. Autoantibodies measured from sera at the diagnosis of T1D in the Finnish Pediatric Diabetes Register. Most common first autoantibody in each genotype is marked bold. Only genotypes with a total frequency of at least five are included.

\begin{tabular}{|c|c|c|c|c|c|}
\hline & \multicolumn{5}{|c|}{ Probable initial autoantibody } \\
\hline & \multicolumn{2}{|c|}{$\begin{array}{l}\text { GADA } \\
\mathrm{N}=96\end{array}$} & \multicolumn{2}{|c|}{$\begin{array}{c}\text { IAA } \\
N=36\end{array}$} & \multirow{2}{*}{$\begin{array}{c}\begin{array}{c}\text { Total } \\
\mathrm{N}=132\end{array} \\
\mathrm{~N}\end{array}$} \\
\hline HLA-genotype & $\mathrm{N}$ & $\%$ & $\mathrm{~N}$ & $\%$ & \\
\hline DR1/10-DQ5/DR1/10-DQ5 & 10 & 83.3 & 2 & 16.7 & 12 \\
\hline DR1/10-DQ5/DR13-DQ6.4 & 10 & 71.4 & 4 & 28,6 & 14 \\
\hline DR1/10-DQ5/DR4-DQ7 & 7 & 53.8 & 6 & 46.2 & 13 \\
\hline DR1/10-DQ5/DR7-DQ2 & 4 & 80.0 & 1 & 20.0 & 5 \\
\hline DR1/10-DQ5/DR5-DQ7 & 7 & 100.0 & 0 & 0.0 & 7 \\
\hline DR1/10-DQ5/DR8-DQ4 & 14 & 70.0 & 6 & 30.0 & 20 \\
\hline DR1/10-DQ5/DR9-DQ9 & 21 & 84.0 & 4 & 16.0 & 25 \\
\hline DR13-DQ6.4/DR8-DQ4 & 3 & 60.0 & 2 & 40,0 & 5 \\
\hline DR13.6.4/DR9-DQ9 & 8 & 100.0 & 0 & 0.0 & 8 \\
\hline DR7-DQ2/DR8-DQ4 & 10 & 55.6 & 8 & 44.4 & 18 \\
\hline DR8-DQ4/DR8-DQ4 & 2 & 40.0 & 3 & 60.0 & 5 \\
\hline
\end{tabular}


This article is protected by copyright. All rights reserved. 
Figure 1

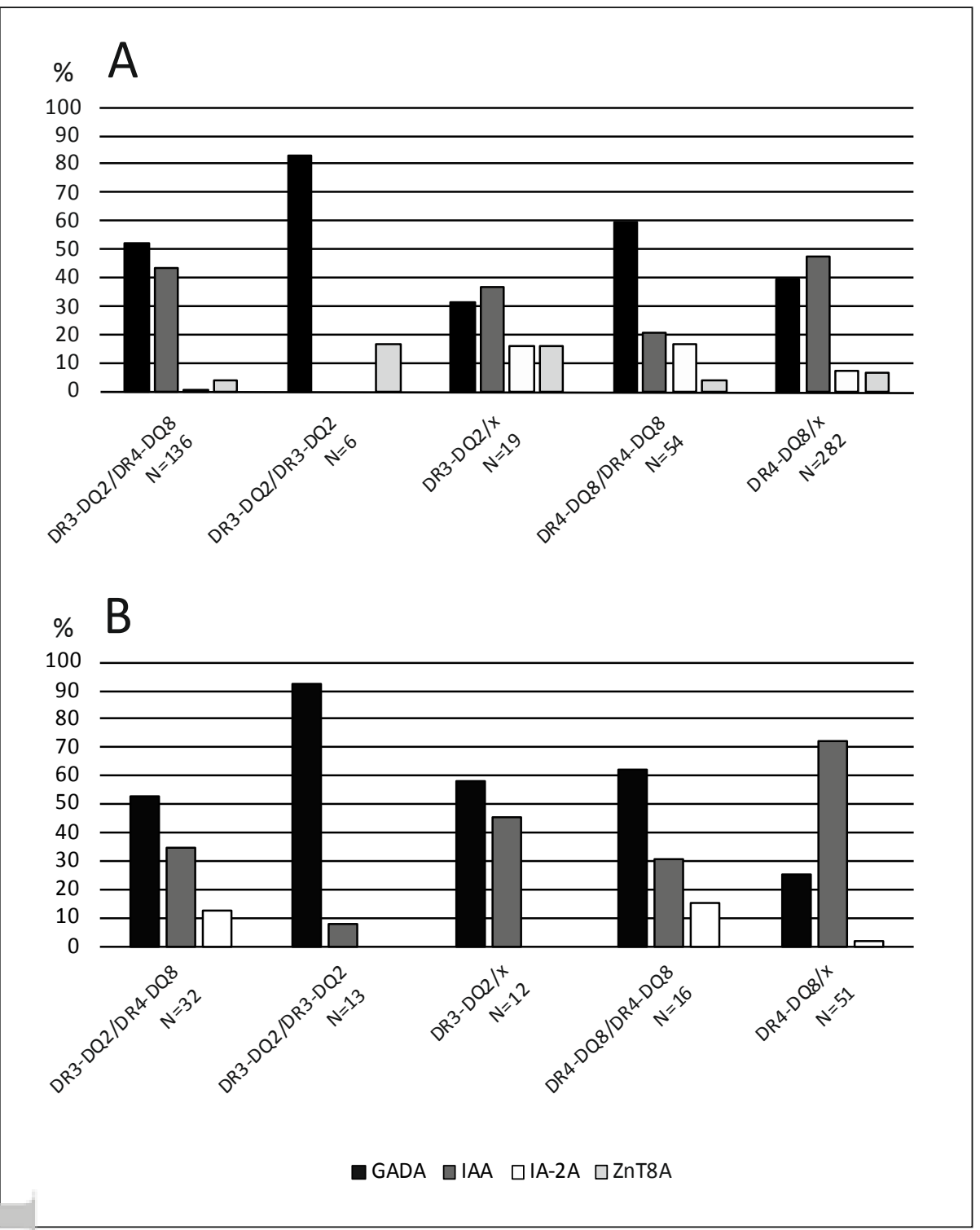

Figure 2

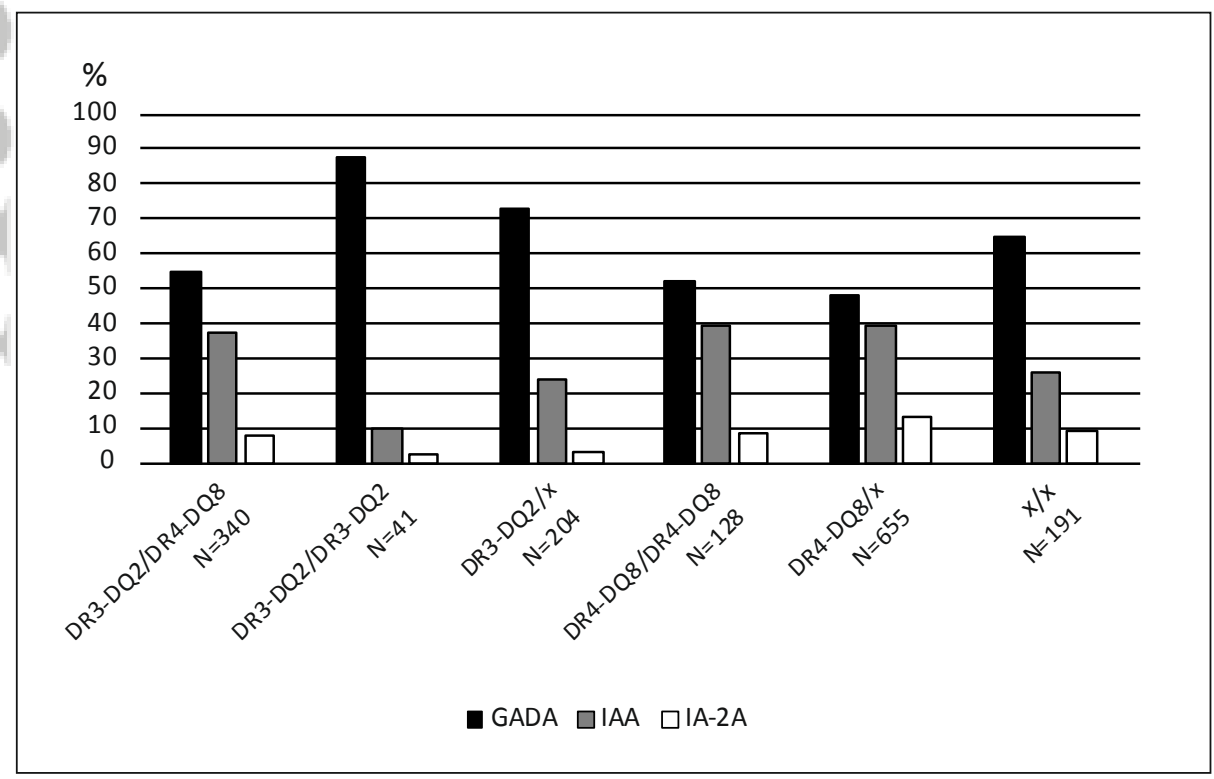

